\title{
Bridging Patients' Needs and Caregivers' Perspectives to Tailor Information Provisioning during Cardiac Rehabilitation
}

\author{
Supraja Sankaran \\ Hasselt University-tUL-Expertise \\ Center for Digital Media \\ Wetenschapspark 2, \\ 3590 Diepenbeek, Belgium \\ supraja.sankaran@uhasselt.be
}

\author{
Cindel Bonneux \\ Hasselt University-tUL \\ Martelarenlaan 42 , \\ 3500 Hasselt, Belgium \\ cindel.bonneux@student.uhasselt.be
}

\author{
Paul Dendale \\ Hasselt University-Faculty of Medicine \\ and Life Sciences; \\ Department of Cardiology, \\ Jessa Hospital, Stadsomvaart 11, \\ 3500 Hasselt, Belgium \\ paul.dendale@uhasselt.be
}

\author{
Karin Coninx \\ Hasselt University-tUL-Expertise \\ Center for Digital Media \\ Wetenschapspark 2, \\ 3590 Diepenbeek, Belgium \\ karin.coninx@uhasselt.be
}

\begin{abstract}
In remote rehabilitation of cardiac patients, patients need a better understanding of various factors influencing their disease condition to become active participants in their care. Nonetheless, current e-learning approaches in healthcare lack personalization and a deeper understanding of individual patient needs. Most e-learning platforms in healthcare are merely an accumulation of content created by caregivers where patients have no means to seek tailored information to suit specific personal needs. This forms a barrier in patient understanding, debilitating them from becoming active stakeholders in their rehabilitation progress. We identify pitfalls in current approaches and gaps in information needs of patients and caregivers' perspectives from literature. We organized two workshops - (i) with various professional caregivers involved in coaching cardiac patients, and (ii) with cardiac patients and their informal caregivers - to bridge caregivers' perspectives with patients' needs. Further, we prototyped and evaluated two tools to support shared decision making of information needs based on outcomes synthesized from the two workshops. In this paper, we discuss results of the workshops and prototype evaluations. Finally, we discuss how this shared decision making approach supports patient understanding and improves their adherence to rehabilitation goals.
\end{abstract}

\section{Patient-centered Computing, E-learning, Shared Decision Making, Cardiac Rehabilitation}

\section{INTRODUCTION}

In cardiac rehabilitation, secondary prevention encompasses measures taken to reduce cardiovascular risks, prevent recurrence of disease and reduce re-hospitalization rates (Mampuya, 2012). A rehabilitation program is typically composed of various elements such as monitoring and managing physiological parameters, physical exercise training, medication intake and adherence, diet, smoking cessation and coaching. A rehabilitation program is typically divided into different phases such as an acute phase in the hospital immediately after a cardiac incident, an ambulatory outpatient phase in a rehabilitation center and a maintenance phase thereafter. With the support of technology and advancements in telemonitoring solutions, remote rehabilitation programs have been successfully implemented. These programs eliminate the need to visit rehabilitation centers by monitoring rehabilitation activities performed in a home environment or outside the hospital-context (Eysenbach, 2005; Klein, Mogles, \& van Wissen, 2011). These technology-assisted rehabilitation approaches have proven to be effective (Frederix et al., 2015; Hansen et al., 2010). Nonetheless, most programs focus exclusively on 
physical exercise training, medication management and progress monitoring (Geraedts, Zijlstra, Zhang, Bulstra, \& Stevens, 2014).

Patients suffering from chronic illness such as cardiovascular diseases often lack a deeper understanding of their condition. Given the evolution towards patient-centered care systems (Krist \& Woolf, 2011), which is especially the case for remote rehabilitation programs, there is a pressing need to enable patients to play an active role in understanding and managing their own disease. A lack of awareness and knowledge of their condition and the progress they are making may result in low adherence and increased drop-out rates over time, reaffirming the law of attrition (Eysenbach, 2005) of e-health technologies. Studies show awareness of and knowledge on one's condition, and the relation with the rehabilitation activities are key to motivate patients and to encourage behavior changes (Balady et al., 2011; Turk-Adawi, Oldridge, Tarima, Stason, \& Shepard, 2013). An extensive review on the factors that influence the information provisioning to cardiac patients emphasized that providing information to patients promotes healthcare decision making, encourages patient's participation in the treatment process, enhances adherence to medical recommendations and prompts modification of health related behavior (Polikandrioti \& Babatsikou, 2013). While the concept of shared decision making is gaining traction, it hasn't been directly applied in the context of e-learning in cardiac rehabilitation yet (Stiggelbout, Pieterse, \& De Haes, 2015).

Typically for a patient, each consultation with a cardiologist is short (with an average of just about 10 minutes per patient), where the patient is provided with a lot of information by caregivers (Elmore et al., 2016). Apart from this overwhelming nature of consultations, patients also have challenges in remembering the information received over a longer duration of time. While multiple systems and studies provide information to patients through educative platforms, and integrated e-learning solutions, there is a clear lack of personalized approaches to information delivery and shared decision making (Holmes-Rovner et al., 2008; Kamphorst, Klein, \& Van Wissen, 2014). These non-personalized approaches with no involvements of patients in the process, again end up overwhelming patients with large amounts of information, which results in low usage of such platforms and does not cater to patients' needs.

In this paper, we contribute by-

(i) Compiling caregivers' perspectives, patients' needs and identifying the similarities and disparities in their perceptions, and

(ii) Synthesizing the expertise of the caregivers and the preferences of patients to provide a tailored collection of information that enhances patient understanding and enables them to become active participants in their care.

We followed a systematic approach (as depicted in Figure 1) to bridge the gaps between caregivers' perspectives and patients' actual information needs by providing the patient with tailored e-learning content in a mobile tele-monitoring application. The approaches undertaken to achieve these are discussed in detail in the subsequent sections of this paper.

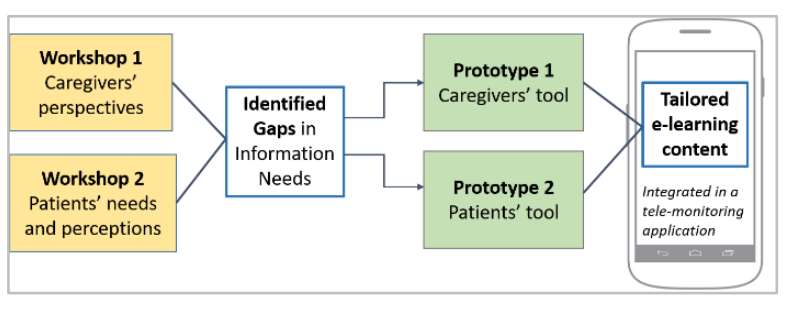

Figure 1: Systematic approach that was used to identify and bridge the gaps between caregivers' perspectives and patient needs.

In the context of this paper, the term 'caregivers' refers to doctors or therapists involved in monitoring and guiding patients through their rehabilitation. 'Informal caregivers' refers to family or friends of patients that are involved in supporting them outside the hospital context.

\section{CURRENT APPROACHES IN E-LEARNING}

To be able to plan and deliver an optimal educational intervention, it is essential for caregivers to have detailed information about what patients already know with respect to cardiac disease and secondary prevention and what their personal information needs are (Brown, Clark, Dalal, Welch, \& Taylor, 2011; Kayaniyil et al., 2009; Scott \& Thompson, 2003). However, multiple studies have shown that healthcare providers are not often aware or inaccurately perceive the educational needs of their patients and patients' characteristics that influence their ability to learn (Brown et al., 2011; Casey, O'Connell, \& Price, 1984; Hagenhoff, Feutz, Conn, Sagehom, \& Moranville-Hunziker, 1994; Karlik \& Yarcheski, 1987; Moynihan, 1984). While healthcare providers are well informed about some patient information needs, they are not always aware of variations in personal preferences. Also, the needs of patients change during various phases of their rehabilitation, and hence, a reassessment of patient needs is necessary (Ghisi et al., 2014). Additionally, a discrepancy has been found between the information that patients desire and their perception of the information that was given by caregivers (Arnetz \& Arnetz, 2009).

Several studies have investigated and reported on disease-related knowledge and information needs 
of cardiac patients (Britto, Ghisi, Motamedi, \& Grace, 2014; Ghisi, Britto, Motamedi, \& Grace, 2015; Scott \& Thompson, 2003; Timmins, 2005; White, Bissell, \& Anderson, 2010). However, according to Timmins, information needs are not necessarily gaps in patients' knowledge that can be solved with education. Rather, they depict what patients desire to receive from their caregivers to cope with their disease (Timmins, 2005).

There is no consensus on what information is most important or needed in the education of cardiac patients. Some trends can be observed in patient information needs in general, but overall these are rather individual and subjective (Timmins, 2005). To cope with these differences between patients, a personalized approach is needed for developing elearning content that can improve patient understanding and enable patients to become active participants in their care. Additionally, tailoring is needed because patients' information needs and disease-related knowledge depend on multiple demographic, clinical and socio-economic variables that can change as they progress through their rehabilitation program (Britto et al., 2014; Ghisi et al., 2015; Greco et al., 2016).

\section{OUR APPROACH TO TAILORING INFORMATION}

To enhance patients' understanding and encourage active participation in their care, we tailor information during their rehabilitation program based on patientspecific needs. However, this information cannot be static during their rehabilitation program. It needs to be updated as patients' understanding improves and as they progress through their rehabilitation.

Patients desire information about multiple broad content categories (e.g. diet, physical activity, medication). However, they often do not want all information about a certain category, but only specific topics adapted to their current knowledge level. In addition, they have individual preferences for the formats in which they would like to receive the information (e.g. a doctor's talk, an animated video, plain text etc.). Considering these preferences of patients, information must be personalized on multiple levels (from high-level content categories to more fine-grained topics) and delivered in specific formats at selected moments of their rehabilitation.

The next sections detail how we identified the gaps in patients' information needs and caregivers' perspectives, and how we bridge them with our systematic approach.

\subsection{Identifying the gaps}

Along with taking into consideration the perspectives of multiple caregivers, it is also important to investigate the information needs of patients and their informal caregivers. Therefore, we conducted workshops with different stakeholders involved in the rehabilitation process to gather their insights on various e-learning content. The details of the workshops are discussed in Table 1.

Table 1: Details of workshops conducted to gain insight into the perspectives of caregivers, patients and informal caregivers on information needs.

\begin{tabular}{|c|c|c|}
\hline & Workshop 1 & Workshop 2 \\
\hline $\begin{array}{l}\text { Key } \\
\text { objective }\end{array}$ & $\begin{array}{l}\text { - Identify } \\
\text { information } \\
\text { relevant for } \\
\text { cardiac } \\
\text { rehabilitation } \\
\text { from caregivers' } \\
\text { perspectives. }\end{array}$ & $\begin{array}{l}\text { - Connect } \\
\text { caregivers' } \\
\text { perspectives from } \\
\text { workshop } 1 \text { with } \\
\text { patients' actual } \\
\text { needs. } \\
\text {-Explore the } \\
\text { changes in } \\
\text { information needs } \\
\text { and preferred } \\
\text { delivery formats } \\
\text { during different } \\
\text { phases of } \\
\text { rehabilitation. }\end{array}$ \\
\hline Participants & $\begin{array}{l}\text { - } 4 \text { caregivers } \\
\text { (a cardiologist, } \\
\text { a psychologist, } \\
\text { a physiotherapist } \\
\text { and a dietician) } \\
\text { - } 2 \mathrm{HCl} \\
\text { researchers }\end{array}$ & $\begin{array}{l}\text { - } 4 \text { cardiac patients } \\
\text { - } 4 \text { informal } \\
\text { caregivers } \\
\text { (1 sibling, } \\
3 \text { partners) } \\
\text { - } 1 \mathrm{HCl} \text { researcher } \\
\text { - } 2 \text { master students } \\
\text { of computer } \\
\text { science }\end{array}$ \\
\hline Materials & \multicolumn{2}{|c|}{$\begin{array}{l}\text { - Printed pre-defined content } \\
\text { - A3 sheets of paper for categorizing } \\
\text { the identified topics into health-related } \\
\text { categories } \\
\text { - Different colored sticky notes per } \\
\text { participant/participant team for writing } \\
\text { down different topics to facilitate in } \\
\text { distinguishing perspectives } \\
\text { - Risk factor tags } \\
\text { - Signs as stickers }\end{array}$} \\
\hline
\end{tabular}

\subsubsection{Process}

In both the workshops, participants were first given a brief introduction and an overview of the objectives and expectations of the workshop. Then each activity was progressively introduced to facilitate an unbiased content generation and not constrain their ideas by subsequent activities. We asked the participants to generate as much content as they could. Some content was pre-defined to generate ideas and provoke discussions during the workshops. For workshop 1 (with caregivers), the pre-defined content was based on standard guidelines, literature and prior experience of participants in making educational videos within their team. This content comprised of 10 content categories and 20 specific topics. For workshop 2 (with patients and their informal caregivers), the outcomes of workshop 1 and some findings from literature were used to come to 10 content 
categories and 39 specific topics. In both workshops, the participants were encouraged to add additional information based on their needs and perspectives. They were allowed to repeat content which were already generated by other participants to gain individual perspectives and identify its relevance from different focal points.

The first four activities of both workshops were similar and were executed in separate groups by the caregivers, patients and informal caregivers. Figure 2 shows an overview of the content put together during these activities.

Activity 1 focused on generating broad content categories. Content categories referred to broad health themes related to cardiac rehabilitation. For example, 'Nutrition', 'Medication', 'Stress and Psychological factors', etc.

In activity 2 specific topics were placed into the various categories that were identified and created in the first activity. Here, participants first began placing the pre-defined topics in the categories. A certain topic could be placed in multiple categories where it is perceived to be conceptually relevant. For example, a topic such as 'Lifestyle changes after an infarction' could be placed in the category of 'Nutrition' as well as 'Physical activity' since patients might have to make lifestyle adaptation both in terms of diet and exercise.

Afterwards, they were encouraged to generate more topics based on their individual experience (for caregivers) and personal needs (for patients and informal caregivers).

In activity 3, participants were asked to map risk factor tags on to the topics. A total of 7 risk factor tags were used, each corresponding to specific modifiable cardiac risk factors (such as reduce cholesterol, manage blood pressure, etc.) that need to be targeted for secondary prevention. These risk factors were derived from a known set of risk factors as defined by the World Heart Federation $[1]$.

Activity 4 was concerned with adding signs to the generated topics. We pre-defined a set of signs for each workshop. For workshop 1 (with the caregivers), visual trigger signs were used to tag the topics and indicate the relation of the topic and the intended behavior change that it should trigger. We used four trigger signs based on persuasion intents that were identified in a study targeting comprehensive tele-rehabilitation for cardiac patients (Sankaran et al., 2016). We represented these triggers using road sign symbols that correspond to a certain way of persuasion. A 'priority' sign $(\gg)$ was used for topics that are critical for a patient to pay more attention to (such

\footnotetext{
${ }^{1}$ https://www.world-heart-federation.org/resources/riskfactors/
}

as, regular check-ups, pursuing rehabilitation goals etc.). A 'stop' sign ( (50P) was used to tag information corresponding to unhealthy behaviors or habits that a patient must be persuaded to stop or avoid (such as, quit smoking, reduce unhealthy diet etc.). A 'go slow' sign (-) was used for aspects that must be undertaken gradually or might be difficult for patients to make a rapid transition or progress (such as, overcoming fear and anxiety, managing stress etc.). Finally, a 'green signal' (G) was used for information that encourages them to pursue certain behaviors and overcome fears or misconceptions (such as, performing certain physical activities, traveling etc.). For workshop 2 (with the patients and informal caregivers), signs were defined based on the perceptions and needs that patients and informal caregivers can have about information. The signs corresponded to 'important' (!), 'difficult to learn' (-)), 'missing in the education curriculum' (Q) and wanting 'to know more' about $(+\mathbf{i})$.

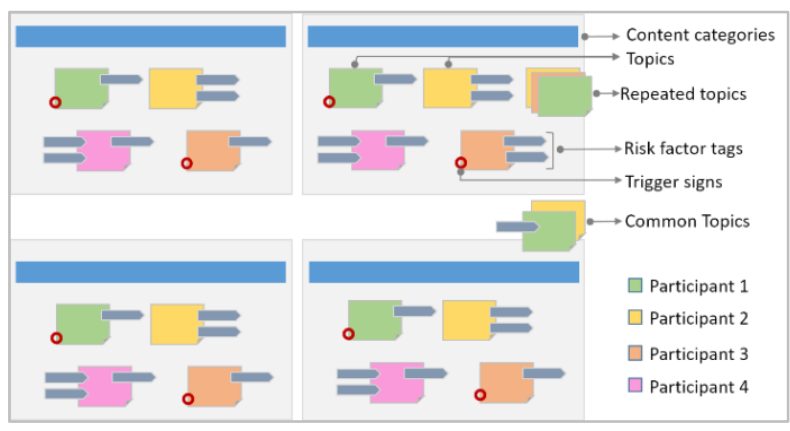

Figure 2: Schematic representation of the outcomes of workshop 1.

In workshop 2 (with the patients and their informal caregivers), two additional activities were executed by the pairs of patients and informal caregivers that came together to the workshop. The patients that were involved in the workshop had completed their rehabilitation program at the hospital and were into their maintenance phase. This selection was specifically made because these patients would have a good understanding of information needs across all phases of rehabilitation.

Activity 5 dealt with the creation of the personal rehabilitation trajectory by using stickers representing different categories. The pairs were asked to describe their information needs by ordering and ranking the categories in time (in the different phases of rehabilitation) and importance.

Lastly, activity 6 investigated patients' and informal caregivers' preferred content delivery formats for receiving information. Delivery format choices included: doctor's talk, patient's talk, peer's talk, animated video, examples, info-graphics and chatbot. Here, the pairs had to label the categories that they used in the trajectory with their preferred 
formats for receiving the information of that category. These could be different depending on the phase in which these were used. Let us consider the example of the topic 'cardiac infarction'. In the first phase of rehabilitation, a patient might like to see an animated video depicting what is a cardiac infarction; while in the maintenance phase, the patient might prefer a video of a doctor talking about how to deal with an infarction.

At the end of these activities, the pairs were asked to orally explain the rehabilitation trajectory they created. An overview of a final outcome of activities 5 and 6 of one pair of workshop 2 is depicted in Figure 3.

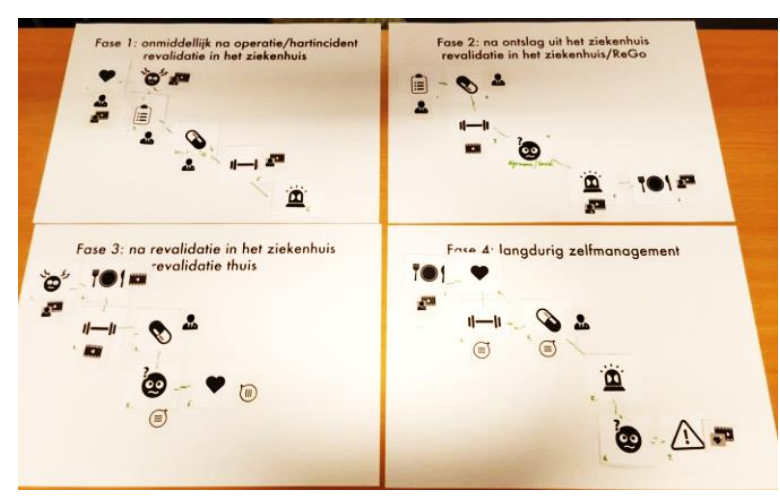

Figure 3: A final outcome of activities 5 and 6 of workshop 2.

\subsubsection{Outcomes}

The outcome of workshop 1 mainly comprised of the information categorization that was put together by the different caregivers. At the end of the workshop, the caregivers ended up with a total of 12 broad content categories and 34 topics spread across the categories with associated risk factor tags and trigger signs. During the workshop, the caregivers emphasized on the importance of taking into consideration the personal condition of the patient when defining the right priorities for topics. Correspondingly, they also expressed the need to be able to adapt the priorities and when to show a certain type of content based on their experience and personal contact with patients.

For workshop 2, there are separate outcomes related to the information categorization and the rehabilitation trajectories. For the information categorization, the patients and informal caregivers did not add additional categories to the 10 predefined ones. However, the patients ended up with a lot more topics spread over the categories than the informal caregivers, 57 and 44 topics respectively. These topics were annotated with risk factor tags and associated signs. For the rehabilitation trajectories, the results mainly comprised of individual trajectories created by patients and their informal caregivers. They then had to give a verbal reasoning behind why certain selections and prioritizations were made. This gave us a more nuanced insight on differences in needs and perceptions of patients and informal caregivers. During the workshop, it became apparent that patients and informal caregivers have different information needs and preferences for content delivery formats based on their experience with their disease and their knowledge.

\subsubsection{Insights}

By comparing the categorizations made by the patients and the informal caregivers with the categorization of the medical staff, it can be observed that the categorizations of both the patients and the informal caregivers are quite different from the categorization of the medical staff. While some topics were clearly classified into a certain category by all participants of a group (i.e. patients and informal caregivers), there were topics that were classified under a different category by different participants of a group. In addition, participants of different workshops placed some topics in the same category, while participants of different groups divided others among different categories. This highlights that different caregivers, patients and informal caregivers can perceive the relevance of similar content differently. Additionally, some topics were perceived difficult to be placed under a single specific category and were therefore placed in-between two contextually related categories.

The key challenge in the workshop with the caregivers came up during the task of mapping behavior triggers when caregivers felt that it is often difficult to generalize such triggers as they largely vary across the patient population. The key challenge during workshop 2 came up during the task of mapping signs (important, missing in the education curriculum, more information wanted and difficult to learn) when it became apparent that the patients and their informal caregivers have different knowledge and information needs. Altogether, the caregivers, the patients and their informal caregivers identified with the need of personalizing e-learning and creating different priorities based on patient needs and risk factors.

As inferred from literature, healthcare providers are often not aware of or inaccurately perceive the educational needs of their patients (Brown et al., 2011; Casey et al., 1984; Ghisi et al., 2014; Hagenhoff et al., 1994; Karlik \& Yarcheski, 1987; Moynihan, 1984). However, this understanding is essential to come to suitable education and coaching of the patient. 


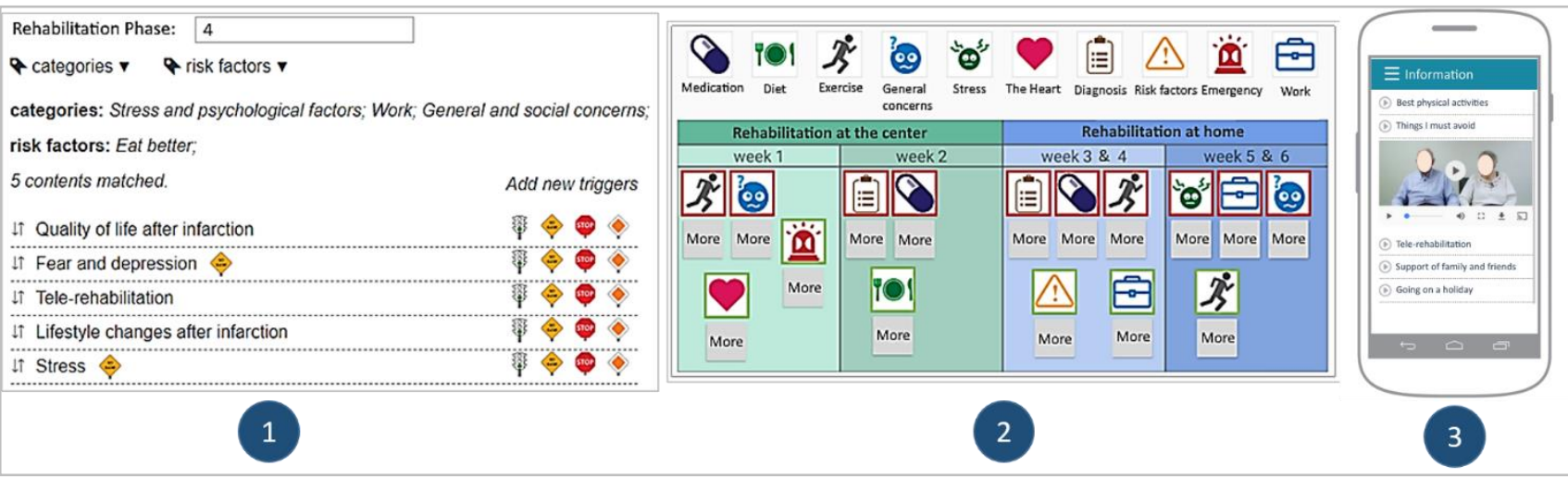

Figure 4: Walkthrough of personalized information provisioning by bridging caregivers' perspectives and patients' needs.

Our proposed approach solves this by giving patients the opportunity to discuss their educational and informational needs with their healthcare providers to come to personalized e-learning content that is tailored to their specific needs. By discussing the needs of the patient during the first encounter between the caregiver and the patient, they both gain insight into the information needs of the patient from the start and use the expertise of the professional caregiver to arrive at e-learning content that is valuable and satisfying for both of them.

\subsection{Bridging the gaps}

The outcomes and insights gained from both workshops were used as inspiration for prototype tools to facilitate a shared decision making process in which a caregiver and a patient can jointly select the e-learning content conforming to medical guidelines and patient needs. The process is constituted of three integrated parts (Figure 4): (1) a desktop-based caregivers' tool, (2) a tablet-based patients' tool that can be used during the first encounter between the caregiver and the patient to gather the patient information needs, and (3) a mobile tele-monitoring application for patients to consume the information during rehabilitation. Each tool is described separately in the following sections.

\subsubsection{Caregivers' tool}

During cardiac rehabilitation, various caregivers are involved in the care and follow-up of the patient. To bring in all these perspectives on the information needs of the patient, caregivers can use the caregivers' tool (Figure 5) to select the information that they perceive as relevant for the rehabilitation of the patient from the perspective of their area of expertise. The content that is selected by the different caregivers is bundled by the tool to provide the patient with a comprehensive set of personalized e-learning content tailored to the patient's condition and risk factor profile.

The caregivers' tool offers multiple opportunities to personalize the e-learning content that is provided to the patient (Figure 5). The tool can be used as follows: (a) the caregiver can filter the exhaustive list of topics based on content categories (e.g. disease-specific information) depending on what the caregiver perceives as most important for the patient in a specific phase. The topics can be filtered based on multiple categories that may be relevant for that phase. Additionally, the filtered topics can be further fine-grained by selecting key risk factors of the patient (e.g. control cholesterol and reduce sugar); (b) On this filtered selection of topics, the caregiver can update the priorities by dragging and dropping topics in a pre-defined order to further tailor it for a patient; (c) Lastly, the caregiver can add or remove signs (e.g. stop, priority, go slow etc.) to specify how the patient should be triggered in the tele-monitoring application to stimulate the necessary behavior change. The caregiver repeats these steps for each of the rehabilitation phases per patient.

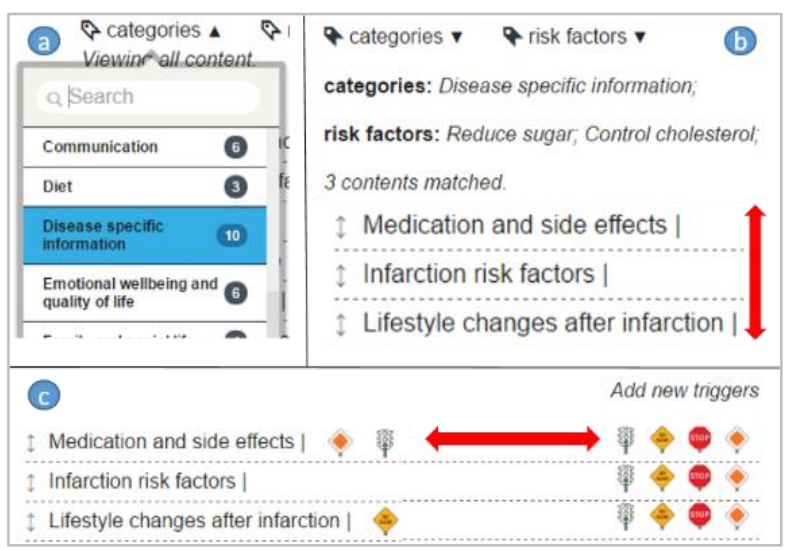

Figure 5: The caregivers' tool can be used by various caregivers to tailor the educational content that is provided to the patient in the tele-monitoring application.

\subsubsection{Patients' tool}

When patients visit professional caregivers for their first consultation, they discuss their information needs with their caregivers and use the patients' tool (Figure 6) to tailor the e-learning content according to their personal needs. In the patients' tool (Figure 6 (1)), there is an overview of the different phases of rehabilitation that the patient will 
go through. The content that was already selected by the caregivers is shown with a red border in the corresponding phases. At the top, the patient can see the broad content categories that are available (e.g. diet, exercise and medication). The patient can add each of these by dragging and dropping them into the desired phases. The categories will then be added with a green border to indicate that the patient added them. Thereafter, the patient can select the specific topics within a category that he seeks more information about (Figure 6 (2)). The topics that were chosen by the caregivers are shown but are disabled to prevent patients from removing those. In other words, some topics are mandated by the caregivers if they are deemed important for the patient. Lastly, the patient can select content delivery formats in which the information will be delivered in the tele-monitoring application (Figure 6 (3)).

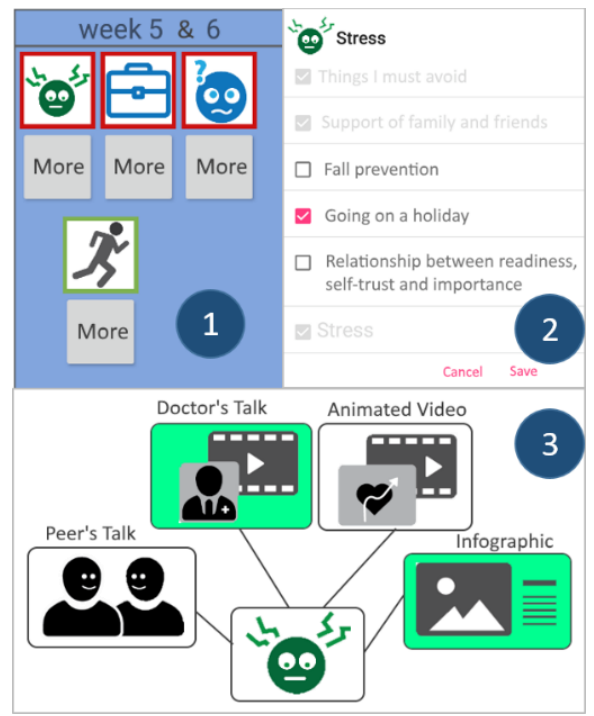

Figure 6: The patients' tool can be used by the patient to tailor the educational content that is provided to the patient in the tele-monitoring application.

\section{EVALUATION}

The implemented prototypes of the two tools were evaluated in two separate user studies with cardiac patients and caregivers. While some usability aspects of the prototypes (such as ease of use, understandability etc.) were also evaluated, the core focus of the studies was to assess the shared decision making process, the relevance of the topics and information presented in each category and determine how different information is perceived by various stakeholders during different phases of rehabilitation.

\subsection{Assessment of patients' tool}

\subsubsection{Process}

The user study for the patient tool was carried out with four cardiac patients (P1 - P4) that were currently rehabilitating at the regional rehabilitation center. The patients received a short introduction to the study and the usage of the tool. Some categories were pre-defined for each rehabilitation phase for every patient by the primary investigators to mimic a real-use scenario. The patients then used the tool to make a selection for their personal e-learning content and preferred content delivery formats. They could add as much information as they wanted and were not restricted in time of using the tool. After they were done with creating their rehabilitation trajectory, the patients filled in a brief questionnaire with statements on the usability and perceived relevance of the tool. They could rate the responses on a 5-point Likert scale, ranging from strongly disagree to strongly agree.

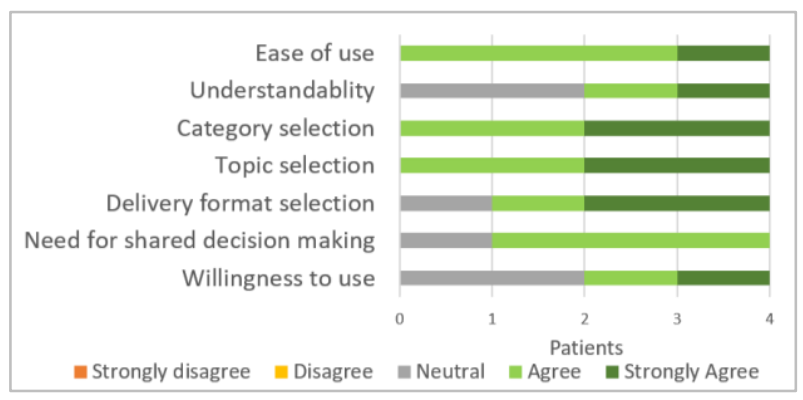

Figure 7: Patients' feedback on usability, usefulness and relevance of using the tool for selecting personalized information during rehabilitation.

\subsubsection{Outcomes}

The trajectories created by each patient were very distinct and no significant similarities could be observed. This suggests that a personalization approach is certainly needed to cater to the personal needs and preferences of patients. The personal preferences and reasons were also emphasized by the comments that patients made while using the tool:

"I will resume my job [after the cardiac incident] in that phase [pointing to a certain phase of rehabilitation]. So, I want to receive information about 'returning to work' before that" [P3].

Contradictory to the assumption that too much information can get overwhelming, P2 selected a lot of categories and topics in the first phase.

"I want as much information as possible [when selecting his/her information for the first phase of rehabilitation]" [P2].

The results of the questionnaire regarding the usability and relevance of the tool are depicted in Figure 7. The patients reported in general that the application was easy to use, intuitive and understandable. Most patients were positive about wanting to be involved in the decision making of their trajectory. This suggests that patients want to be active participants in their care and rehabilitation 
process and an approach like this is needed to offer patients these opportunities.

\subsection{Assessment of caregivers' tool}

\subsubsection{Process}

To investigate caregivers' perspectives on the proposed approach, the usability and relevance of the proposed content of the caregivers' tool were evaluated in a separate user study with four caregivers (a physiotherapist, a dietician, a psychologist and a cardiac nurse involved in cardiac rehabilitation). Apart from the psychologist, none of them were involved in the initial caregiver workshop.

The caregivers received a short introduction to the purpose of the study and how to use the tool. Afterwards, they were given three use cases in which cardiac patient personas and their risk factors were described. The caregivers were asked to use the tool to select the e-learning content that they considered as relevant for the different personas across different phases of rehabilitation and elaborate orally on why they selected the specific content. They were also allowed to just go over the available topics and reflect on their relevance. Given the busy schedules of the caregivers, the duration of the study was limited by only letting them consider the categories and risk factors that are relevant from the perspective of their specialization (e.g. physiotherapists looking at aspects of physical activity, dietician looking at aspects of diet and nutrition etc.).

The caregivers were asked to consider the topics in the categories and the associated risk factors and reflect upon the relevance and correctness of the topics within each category or risk factor. They were also asked to reflect on default priorities and trigger signs for each topic within a category. Suggestions for improvements were written down and can be used as input for further improvements of the tool. After using the tool, caregivers were asked to rank some statements regarding the usability and the relevance of the content on a 5point Likert scale using a short questionnaire.

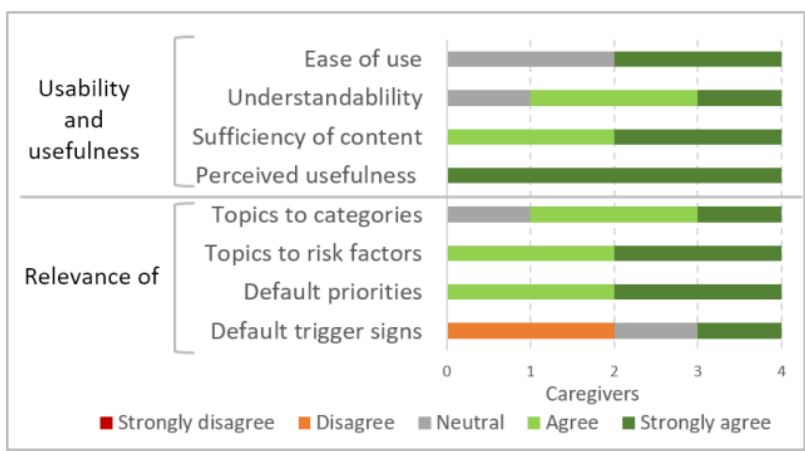

Figure 8: Caregivers' perspectives on the usability, usefulness and relevance of content presented in the tool.

\subsubsection{Outcomes}

The results of the questionnaire regarding the usability and relevance of the content of the tool are depicted in Figure 8. Some caregivers found the tool easier to use than others, but no negative scores were given on the usability and comprehensiveness of the tool.

All caregivers perceived the topics and categories that are available in the tool as sufficient. The scores for the default priorities of the topics are very positive and are divided equally between agree and strongly agree. However, for the default trigger signs the scores are rather low, with two values of disagree and one of neutral. Caregivers opined that the behavior triggers are highly dependent on an individual and it is not always feasible to assign defaults.

\begin{abstract}
"The behavioral triggers have to cater to different motivational frames of reference which is different for different patients. We also cannot fix a single motivational frame across different stages of rehabilitation" [Psychologist]
\end{abstract}

All caregivers were unanimously convinced that providing a filtered set of content to the patients is a suitable approach. Additionally, caregivers expressed that they are willing to use such a tool in their regular practice for selecting personalized elearning content for patients. They liked that the tool offers them the opportunity to tailor information for various patients in a structured systematic manner.

\section{DISCUSSION}

Current e-learning systems overwhelm patients with large amounts of information that is not tailored to their individual needs and preferences. Therefore, these systems do not have the intended effects on patient understanding and behavior change. This prompted us to investigate the gaps in patient information needs and caregivers' perspectives.

\subsection{Reflection on outcomes}

The need for personalization was expressed during the workshop with caregivers by all the participants and became apparent when analyzing the results of the workshop with cardiac patients and their informal caregivers.

Although the focus of the user studies was not on usability and rather on assessing the suitability of the proposed approach and the relevance of the available content in the tool, we can conclude that in general the tools are comprehensive and easy to use. The process of shared decision making by the use of proposed tools were deemed simple to be used in real practice and consultations.

In general, the results of both studies were positive and can be considered as encouragement to further 
investigate our proposed approach of tailoring elearning content in a shared decision making process with involvement of caregivers and patients.

\subsection{Reflection on contributions}

As mentioned in the introduction, the contributions highlighted in this paper are -

(i) Compiling caregivers' perspectives, patients' needs and identifying the similarities and disparities in their perceptions, and

(ii) Synthesizing the expertise of the caregivers and the preferences of patients to provide a tailored collection of information that enhances patient understanding and enables them to become active participants in their care.

By reiterating our contributions and insights gained from the outcomes of our approach, we hope to inspire other $\mathrm{HCl}$ researchers to adopt a similar approach to assess the impact of tailored information provisioning on other health domains as well.

During the workshop with the patients and their informal caregivers, some patient participants mentioned that they ask their caregivers about the possible side-effects of medication, if the medication is really needed and to reduce the amount of medication that they have to take. This suggests that those patients are initiating the shared decision making process themselves, even without being offered this possibility explicitly by caregivers. This is conforming to the recent trends towards patientcentered care and shared decision making. However, during the user tests, the participating patients were less proactive in terms of voluntarily seeking information but were willing to use a similar tool. Being involved in the decision making process is rather personal and subjective and therefore patients' desires regarding this can be influenced by personal characteristics and preferences. Although most (but not all) participants of the patient study wanted to be involved in a shared decision making process, the caregivers must assess at the beginning of the encounter if the patient wants to be an active participant in the rehabilitation process and provide support accordingly. If patients are not willing to be involved in the decision making, they can still benefit from our proposed approach since the tailoring from the caregivers' side (Figure 5) already increases the relevance of the proposed elearning content to the patient.

All caregivers were enthusiastic about the proposed approach and were willing to use a similar tool in their regular practice. This emphasizes that caregivers are willing to incorporate patients' opinion in the decision making process by offering them the opportunity to tailor e-learning content to their needs. They are all convinced that offering patients a selection of content is valuable and will benefit the
patient.The experience of caregivers already gives an indication on the feasibility and possible effectiveness of our approach.

\subsection{Future directions}

One open challenge that remains is defining suitable content for each topic and determining relevant behavior triggers for each patient. It is difficult to generalize this for all patients, so tailoring of content in a topic and adapting the behavior triggers is essential. Caregivers suggested that the risk factor profiles of patients can be used as a reference framework for defining these appropriate behavior triggers. This suggestion can form a starting point for developing tele-monitoring applications to trigger long-term behavior change effectively. Tailored information provisioning and shared decision making by using tools similar to the prototypes proposed in this paper could be adopted in holistic, comprehensive rehabilitation approaches to assess its effectiveness in increasing patient motivation and triggering behavior change. Incorporating such methods in other comprehensive rehabilitation programs can also give insights on the impact of such approaches on rehabilitation and health outcomes.

\section{CONCLUSION}

To enhance patients' understanding and enable them to become active participants in their care, we propose to tailor the e-learning content that is delivered to the patients based on their needs. We identified the gaps between caregivers' perspectives and actual patient needs and proposed an approach to bridge these effectively by providing the patient and the caregiver the opportunity to tailor e-coaching content to patient needs. An integrated prototype consisting of three parts (caregivers' tool, patients' tool and telemonitoring application with tailored e-learning content) was developed to illustrate the proposed approach of a personalized e-learning trajectory conforming to medical expertise and patient needs and evaluated in two separate user studies. All participants were positive about the personalization and systematic way of working. Patients expressed the desire of wanting to be active participants in their own care, which was recognized and affirmed by the caregivers. This opens up new possibilities for future solutions building upon this approach to tailor e-learning and coaching of patients in cardiac rehabilitation to patient needs.

\section{REFERENCES}

Arnetz, J., E. , \& Arnetz, B., B. (2009). Gender Differences in Patient Perceptions of Involvement in Myocardial Infarction Care. 
European Journal of Cardiovascular Nursing, 8(3), 174-181.

Balady, G. J., Ades, P. A., Bittner, V. A., Franklin, B. A., Gordon, N. F., Thomas, R. J., ... Yancy, C. W. (2011). Referral, Enrollment, and Delivery of Cardiac Rehabilitation/Secondary Prevention Programs at Clinical Centers and Beyond: A Presidential Advisory From the American Heart Association. Circulation, 124(25), 2951-2960.

Britto, R., Ghisi, G. L. M., Motamedi, N., \& Grace, S. (2014). PM486 Socio-demographic correlates of disease-related knowledge in cardiac rehabilitation participants in Toronto, Canada (Vol. 9).

Brown, J. P., Clark, A. M., Dalal, H., Welch, K., \& Taylor, R. S. (2011). Patient education in the management of coronary heart disease. Cochrane Database of Systematic Reviews(12).

Casey, E., O'Connell, J. K., \& Price, J. H. (1984). Perceptions of educational needs for patients after myocardial infarction. Patient Education and Counseling, 6(2), 77-82.

Elmore, N., Burt, J., Abel, G., Maratos, F. A., Montague, J., Campbell, J., \& Roland, M. (2016). Investigating the relationship between consultation length and patient experience: a cross-sectional study in primary care. British Journal of General Practice, 66(653), e896e903.

Eysenbach, G. (2005). The Law of Attrition. J Med Internet Res, 7(1), e11.

Frederix, I., Van Driessche, N., Hansen, D., Berger, J., Bonne, K., Alders, T., \& Dendale, P. (2015). Increasing the medium-term clinical benefits of hospital-based cardiac rehabilitation by physical activity telemonitoring in coronary artery disease patients. European Journal of Preventive Cardiology, 22(2), 150-158.

Geraedts, H. A., Zijlstra, W., Zhang, W., Bulstra, S., \& Stevens, M. (2014). Adherence to and effectiveness of an individually tailored homebased exercise program for frail older adults, driven by mobility monitoring: design of a prospective cohort study. BMC Public Health, 14(1), 570.

Ghisi, G. L. M., Britto, R., Motamedi, N., \& Grace, S. L. (2015). Disease-related knowledge in cardiac rehabilitation enrollees: Correlates and changes. Patient Education and Counseling, 98(4), 533-539.

Ghisi, G. L. M., Grace, S. L., Thomas, S., Evans, M. F., Sawula, H., \& Oh, P. (2014). Healthcare providers' awareness of the information needs of their cardiac rehabilitation patients throughout the program continuum. Patient Education and Counseling, 95(1), 143-150.
Greco, A., Cappelletti, E. R., Monzani, D., Pancani, L., D'Addario, M., Magrin, M. E., . . . Steca, P. (2016). A longitudinal study on the information needs and preferences of patients after an acute coronary syndrome. BMC Family Practice, 17(1), 136.

Hagenhoff, B. D., Feutz, C., Conn, V. S., Sagehom, K. K., \& Moranville-Hunziker, M. (1994). Patient education needs as reported by congestive heart failure patients and their nurses. Journal of Advanced Nursing, 19(4), 685-690.

Hansen, D., Dendale, P., Raskin, A., Schoonis, A., Berger, J., Vlassak, I., \& Meeusen, R. (2010). Long-term effect of rehabilitation in coronary artery disease patients: Randomized clinical trial of the impact of exercise volume. Clinical Rehabilitation, 24(4), 319-327.

Holmes-Rovner, M., Stommel, M., Corser, W. D., Olomu, A., Summers Holtrop, J., Siddiai, A., \& Dunn, S. L. (2008). Does Outpatient Telephone Coaching Add to Hospital Quality Improvement Following Hospitalization for Acute Coronary Syndrome? Journal of General Internal Medicine, 23(9), 1464-1470.

Kamphorst, B. A., Klein, M. C. A., \& Van Wissen, A. (2014). Autonomous e-coaching in the wild: empirical validation of a model-based reasoning system. Paper presented at the Proceedings of the 2014 international conference on Autonomous agents and multi-agent systems, Paris, France.

Karlik, B. A., \& Yarcheski, A. (1987). Learning needs of cardiac patients: a partial replication study. Heart \& lung: the journal of critical care, 16(5), 544-551.

Kayaniyil, S., Ardern, C. I., Winstanley, J., Parsons, C., Brister, S., Oh, P., . . G Grace, S. L. (2009). Degree and Correlates of Cardiac Knowledge and Awareness among Cardiac Inpatients. Patient Education and Counseling, 75(1), 99107.

Klein, M., Mogles, N., \& van Wissen, A. (2011). Why Won't You Do What's Good for You? Using Intelligent Support for Behavior Change, Berlin, Heidelberg.

Krist, A. H., \& Woolf, S. H. (2011). A Vision for Patient-Centered Health Information Systems. JAMA, 305(3), 300-301.

Mampuya, W. M. (2012). Cardiac rehabilitation past, present and future: an overview. Cardiovascular Diagnosis and Therapy, 2(1), 3849.

Moynihan, M. (1984). Assessing the educational needs of post-myocardial infarction patients. The Nursing clinics of North America, 19(3), 441-447. 
Polikandrioti, M., \& Babatsikou, F. (2013). Information to coronary disease patients. Health Science Journal, 7(1), 3-10.

Sankaran, S., Frederix, I., Haesen, M., Dendale, P., Luyten, K., \& Coninx, K. (2016). A Grounded Approach for Applying Behavior Change Techniques in Mobile Cardiac TeleRehabilitation. Paper presented at the Proceedings of the 9th ACM International Conference on PErvasive Technologies Related to Assistive Environments, Corfu, Island, Greece.

Scott, J. T., \& Thompson, D. R. (2003). Assessing the information needs of post-myocardial infarction patients: a systematic review. Patient Education and Counseling, 50(2), 167-177.

Stiggelbout, A. M., Pieterse, A. H., \& De Haes, J. C. J. M. (2015). Shared decision making: Concepts, evidence, and practice. Patient Education and Counseling, 98(10), 1172-1179.

Timmins, F. (2005). A review of the information needs of patients with acute coronary syndromes. Nursing in Critical Care, 10(4), 174183.

Turk-Adawi, K. I., Oldridge, N. B., Tarima, S. S., Stason, W. B., \& Shepard, D. S. (2013). Cardiac Rehabilitation Patient and Organizational Factors: What Keeps Patients in Programs? Journal of the American Heart Association: Cardiovascular and Cerebrovascular Disease, 2(5), e000418.

White, S., Bissell, P., \& Anderson, C. (2010). Patients' Perspectives on Cardiac Rehabilitation, Lifestyle Change and Taking Medicines: Implications for Service Development. Journal of Health Services Research \& Policy, 15(2_suppl), 47-53. 